\title{
Weak lensing of the CMB: Cumulants of the probability distribution function
}

\author{
Michael Kesden, Asantha Cooray, and Marc Kamionkowski \\ Theoretical Astrophysics, California Institute of Technology, Pasadena, California 91125
}

(Received 16 August 2002; published 29 October 2002)

\begin{abstract}
We discuss the real-space moments of temperature anisotropies in the cosmic microwave background (CMB) due to weak gravitational lensing by intervening large-scale structure. We show that if the probability distribution function of primordial temperature anisotropies is Gaussian, then it remains unchanged after gravitational lensing. With finite resolution, however, nonzero higher-order cumulants are generated both by lensing autocorrelations and by cross-correlations between the lensing potential and secondary anisotropies in the CMB such as the Sunayev-Zel'dovich (SZ) effect. Skewness is produced by these lensing-SZ correlations, while kurtosis receives contributions from both lensing alone and lensing-SZ correlations. We show that if the projected lensing potential is Gaussian, all cumulants of higher order than the kurtosis vanish. While recent results raise the possibility of detection of the skewness in upcoming data, the kurtosis will likely remain undetected.
\end{abstract}

DOI: 10.1103/PhysRevD.66.083007

PACS number(s): 98.70.Vc, 98.35.Ce, 98.80.Es

\section{INTRODUCTION}

Weak gravitational lensing deflects the paths of cosmic microwave background (CMB) photons propagating from the surface of last scattering. One result of this lensing is the transfer of power from large angular scales associated with acoustic-peak structures to small angular scales in the damping tail of the anisotropy power spectrum $[1,2]$. This transfer only results in a few-percent modification of the power associated with the acoustic-peak structure, and the increase in power along the damping tail is significantly smaller than that generated by secondary anisotropies due to reionization [3]. To identify the effect of gravitational lensing on CMB data, it is necessary to consider signatures beyond that in the angular power spectrum of temperature fluctuations. The existence of nonvanishing higher order cumulants is one such non-Gaussian signature lensing can generate.

Since gravitational lensing conserves surface brightness, CMB fluctuations from lensing are at the second order in temperature fluctuations and result in non-Gaussian behavior through non-linear mode coupling. Though lensing alone does not lead to a three-point correlation function, the correlation between lensing and other secondary anisotropies can lead to such a contribution. This three-point correlation has been widely discussed in the literature in terms of its Fourierspace analogue, the bispectrum [4]. Weak lensing of the primary anisotropies can produce a four-point correlation due to its non-linear mode-coupling nature [5-7], as can correlations between lensing and secondary effects [7]. When probed appropriately through quadratic statistics such as the power spectrum of the squared-temperature map, the trispectrum due to lensing alone can be used for a modelindependent recovery of the projected mass distribution out to the last scattering surface $[8,9]$. Though these statistics have been shown to be interesting and potentially detectable, measurement of these Fourier-based statistics is challenging and techniques are still underdeveloped for this purpose.

Here, we discuss real-space moments of the lensed CMB temperature anisotropies. Real-space statistics are easily measurable from data. The only drawbacks are that they are unlikely to be optimal and only provide limited knowledge of the full non-Gaussian aspect of the temperature distribution. The first attempts to measure non-Gaussianity in the Cosmic Background Explorer (COBE) data relied on realspace cumulants [10], as will attempts using data from its successor experiments such as Microwave Anisotropy Probe (MAP) and Planck. This motivates our emphasis here on the real-space cumulants such as the skewness and kurtosis; we make several remarks on higher-order cumulants as well. As part of this calculation, we extend a previous discussion of the kurtosis due to lensing in Ref. [5] and also consider effects related to correlations between lensing and secondary effects such as the Sunyaev-Zel'dovich [(SZ) [11]] effect.

Real-space moments can be derived from the one-point probability distribution function (PDF) of temperature fluctuations, and can conversely be used to constrain the form of this function. In the case of infinite angular resolution, we conclude that lensing does not modify the PDF of temperature anisotropies produced at the last scattering surface, which is a reflection on the fact that lensing does not create new power but rather transfers power from large to small angular scales. The higher-order moments are only generated in a temperature map by finite-resolution effects such as beam smoothing introduced either experimentally or artificially by explicit filtering.

The paper is organized as follows. In Sec. II, we introduce formalism concerning the weak-lensing approximation and define the bispectrum, trispectrum, and corresponding higher-order quantities. The bispectrum and trispectrum induced in the CMB by lensing and secondary anisotropies are derived in Sec. III, and some remarks are made concerning higher-order cumulants as well. The nonzero bispectrum and trispectrum yield a skewness and kurtosis, respectively, in the one-point distribution function of the CMB as shown in Sec. IV. We refer the reader to Ref. [9] for additional details related to the effect of lensing on CMB anisotropies. Though we present a general discussion, we illustrate our results in Sec. V using the currently favored cold dark matter with a cosmological constant model $(\Lambda \mathrm{CDM})$ with $\Omega_{b}=0.05, \Omega_{m}$ $=0.35, \Omega_{\Lambda}=0.65, h=0.65$ and $\sigma_{8}=0.9$. Results for a 
model with $\sigma_{8}=1.2$ as suggested by the Cosmic Background Imager (CBI) are also considered.

\section{LENSING CONTRIBUTION TO CMB FLUCTUATIONS}

In order to derive the effects of weak lensing on the CMB, we follow Refs. [2,6] and adopt a flat sky approximation. As discussed in prior papers $[2,4]$, weak lensing remaps temperature through angular deflections along the photon path:

$$
\begin{aligned}
\widetilde{\Theta}(\hat{\mathbf{n}})= & \Theta(\hat{\mathbf{n}}+\nabla \phi) \\
= & \Theta(\hat{\mathbf{n}})+\nabla_{i} \phi(\hat{\mathbf{n}}) \nabla^{i} \Theta(\hat{\mathbf{n}}) \\
& +\frac{1}{2} \nabla_{i} \phi(\hat{\mathbf{n}}) \nabla_{j} \phi(\hat{\mathbf{n}}) \nabla^{i} \nabla^{j} \Theta(\hat{\mathbf{n}})+\ldots
\end{aligned}
$$

Here, $\Theta(\hat{\mathbf{n}})$ is the unlensed primary component of the CMB in direction $\hat{\mathbf{n}}$ at the last-scattering surface, $\widetilde{\Theta}(\hat{\mathbf{n}})$ is the lensed map, $\phi(\hat{\mathbf{n}})$ is the projected gravitational potential, and $\nabla \phi$ is the lensing deflection angle. It should be understood that in the presence of low-redshift contributions to CMB fluctuations resulting from large-scale structure, the total map includes secondary contributions which we denote by $\Theta^{\mathrm{s}}(\hat{\mathbf{n}})$. Since the weak-lensing deflection angles $\nabla \phi$ also trace the large-scale structure at low redshifts, secondary effects which are first order in density fluctuations correlate with the lensing deflection angles. These secondary effects include the integrated Sachs-Wolfe [(SW) [12]] and the SZ [11] effects [4]. In all real cases, a noise component denoted by $\Theta^{\mathrm{n}}(\hat{\mathbf{n}})$ due to finite experimental sensitivity must be included as well. Thus the total observed CMB anisotropy will be $\Theta^{\mathrm{t}}(\hat{\mathbf{n}})=\widetilde{\Theta}(\hat{\mathbf{n}})+\Theta^{\mathrm{s}}(\hat{\mathbf{n}})+\Theta^{\mathrm{n}}(\hat{\mathbf{n}})$. In the following discussion, secondary anisotropies $\Theta^{\mathrm{s}}(\hat{\mathbf{n}})$ will be neglected until Sec. III B while the effects of instrumental noise $\Theta^{\mathrm{n}}(\hat{\mathbf{n}})$ on the PDF are discussed in Sec. IV.

Taking the Fourier transform, as appropriate for a flat sky, we write

$$
\begin{aligned}
\widetilde{\Theta}(\mathbf{l}) & =\int d \hat{\mathbf{n}} \widetilde{\Theta}(\hat{\mathbf{n}}) e^{-i \mathbf{l} \cdot \hat{\mathbf{n}}} \\
& =\Theta(\mathbf{l})-\int \frac{d^{2} \mathbf{l}^{\prime}}{(2 \pi)^{2}} \Theta\left(\mathbf{l}^{\prime}\right) L\left(\mathbf{l}, \mathbf{l}^{\prime}\right),
\end{aligned}
$$

where

$$
\begin{aligned}
L\left(\mathbf{l}, \mathbf{l}^{\prime}\right)= & \phi\left(\mathbf{l}-\mathbf{l}^{\prime}\right)\left[\left(\mathbf{l}-\mathbf{l}^{\prime}\right) \cdot \mathbf{l}^{\prime}\right]+\frac{1}{2} \int \frac{d^{2} \mathbf{l}^{\prime \prime}}{(2 \pi)^{2}} \phi\left(\mathbf{l}^{\prime \prime}\right) \\
& \times \phi^{*}\left(\mathbf{l}^{\prime \prime}+\mathbf{l}^{\prime}-\mathbf{l}\right)\left(\mathbf{l}^{\prime \prime} \cdot \mathbf{l}^{\prime}\right)\left[\left(\mathbf{l}^{\prime \prime}+\mathbf{l}^{\prime}-\mathbf{l}\right) \cdot \mathbf{l}^{\prime}\right]+\ldots
\end{aligned}
$$

We define the power spectrum, bispectrum, trispectrum and the $n$-point correlator in Fourier space in the usual way:

$$
\begin{aligned}
&\left\langle\widetilde{\Theta}\left(\mathbf{l}_{1}\right) \widetilde{\Theta}\left(\mathbf{l}_{2}\right)\right\rangle=(2 \pi)^{2} \delta_{\mathrm{D}}\left(\mathbf{l}_{12}\right) \widetilde{C}_{l_{1}}^{\Theta}, \\
&\left\langle\widetilde{\Theta}\left(\mathbf{l}_{1}\right) \ldots \widetilde{\Theta}\left(\mathbf{l}_{3}\right)\right\rangle_{c}=(2 \pi)^{2} \delta_{\mathrm{D}}\left(\mathbf{l}_{123}\right) \widetilde{B}^{\Theta}\left(\mathbf{l}_{1}, \mathbf{l}_{2}, \mathbf{l}_{3}\right), \\
&\left\langle\widetilde{\Theta}\left(\mathbf{l}_{1}\right) \ldots \widetilde{\Theta}\left(\mathbf{l}_{4}\right)\right\rangle_{c}=(2 \pi)^{2} \delta_{\mathrm{D}}\left(\mathbf{l}_{1234}\right) \widetilde{T}^{\Theta}\left(\mathbf{l}_{1}, \mathbf{l}_{2}, \mathbf{l}_{3}, \mathbf{l}_{4}\right), \\
&\left\langle\widetilde{\Theta}\left(\mathbf{l}_{1}\right) \ldots \widetilde{\Theta}\left(\mathbf{l}_{n}\right)\right\rangle_{c}=(2 \pi)^{2} \delta_{\mathrm{D}}\left(\mathbf{l}_{1} \ldots n\right) \widetilde{T}_{n}^{\Theta}\left(\mathbf{l}_{1}, \ldots, \mathbf{l}_{n}\right) .
\end{aligned}
$$

where $\mathbf{l}_{1} \ldots n \equiv \mathbf{l}_{1}+\ldots+\mathbf{l}_{n}$, and the subscript $c$ denotes the connected portion of the correlation function. We make the assumption that primary anisotropies at the last scattering surface are Gaussian implying that all cumulants higher than the power spectrum vanish: $\left\langle\Theta\left(\mathbf{l}_{1}\right) \ldots \Theta\left(\mathbf{l}_{n}\right)\right\rangle_{c}=0$, when $n$ $>2$.

The power spectra for lensing autocorrelations and lensing-secondary cross-correlations are defined analogously:

$$
\begin{gathered}
\left\langle\phi\left(\mathbf{l}_{1}\right) \phi\left(\mathbf{l}_{2}\right)\right\rangle=(2 \pi)^{2} \delta_{\mathrm{D}}\left(\mathbf{l}_{12}\right) C_{l_{1}}^{\phi \phi}, \\
\left\langle\phi\left(\mathbf{l}_{1}\right) \Theta^{\mathrm{s}}\left(\mathbf{l}_{2}\right)\right\rangle=(2 \pi)^{2} \delta_{\mathrm{D}}\left(\mathbf{l}_{12}\right) C_{l_{1}}^{\phi \mathrm{s}} .
\end{gathered}
$$

Primary CMB anisotropies $\Theta(\hat{\mathbf{n}})$ are generated at the surface of last scatter at $z \simeq 1,100$, while the lensing potential $\phi(\hat{\mathbf{n}})$ and secondary contributions $\Theta^{\mathrm{s}}(\hat{\mathbf{n}})$ arise from large-scale structure at much lower redshifts $(z \simeq 3)$. As such, correlations between these quantities vanish: $\left\langle\Theta\left(\mathbf{l}_{1}\right) \phi\left(\mathbf{l}_{2}\right)\right\rangle$ $=\left\langle\Theta\left(\mathbf{l}_{1}\right) \Theta^{\mathrm{s}}\left(\mathbf{l}_{2}\right)\right\rangle=0$.

The $n$th cumulant of the temperature anisotropies is defined in the usual manner,

$$
\begin{aligned}
C^{n}(\theta)= & \int \frac{d^{2} \mathbf{l}_{1}}{(2 \pi)^{2}} \ldots \frac{d^{2} \mathbf{l}_{n}}{(2 \pi)^{2}}\left\langle\Theta^{\mathrm{t}}\left(\mathbf{l}_{1}\right) \ldots \Theta^{\mathrm{t}}\left(\mathbf{l}_{n}\right)\right\rangle_{c} \\
& \times W\left(l_{1} \theta\right) \ldots W\left(l_{n} \theta\right),
\end{aligned}
$$

where $\theta$ is the smoothing scale of the map from which the cumulants are determined, and $W(l \theta)$ is the smoothing window function. We will use Gaussian window functions throughout this paper. In general, the finite resolution of real CMB anisotropy experiments induces Gaussian smoothing at the angular scale of the experimental beam size. For infinite resolution, we take $\theta \rightarrow 0$ such that $W(l \theta) \rightarrow 1$. The variance, skewness, and kurtosis defined later in this paper can all be expressed in terms of cumulants

$$
\sigma^{2}(\theta)=C^{2}(\theta), \quad S(\theta)=\frac{C^{3}(\theta)}{\left[C^{2}(\theta)\right]^{3 / 2}}, \quad K(\theta)=\frac{C^{4}(\theta)}{\left[C^{2}(\theta)\right]^{2}} .
$$

\section{POWER SPECTRUM, BISPECTRUM AND TRISPECTRUM}

Using the formalism introduced in the previous section, we can calculate the moments of the CMB fluctuations generated by lensing assuming Gaussian fluctuations at the surface of last scatter. Because $\phi$ is a small parameter, terms beyond linear order in $C_{l}^{\phi \phi}$ are neglected in these calculations. The power spectrum for the lensed map is $[1,2]$ 


$$
\begin{aligned}
\widetilde{C}_{l}^{\Theta}= & {\left[1-\int \frac{d^{2} \mathbf{l}_{1}}{(2 \pi)^{2}} C_{l_{1}}^{\phi \phi}\left(\mathbf{l}_{1} \cdot \mathbf{l}\right)^{2}\right] C_{l}^{\Theta} } \\
& +\int \frac{d^{2} \mathbf{l}_{1}}{(2 \pi)^{2}} C_{\left|\mathbf{l}-\mathbf{l}_{1}\right|}^{\Theta} C_{l_{1}}^{\phi \phi}\left[\left(\mathbf{l}-\mathbf{l}_{1}\right) \cdot \mathbf{l}_{1}\right]^{2} .
\end{aligned}
$$

The variance, or the second moment of the temperature, can be obtained following Eq. (6):

$$
\sigma^{2}(\theta)=\int \frac{d^{2} \mathbf{l}}{(2 \pi)^{2}} \widetilde{C}_{l}^{\Theta} W^{2}(l \theta) .
$$

Substituting Eq. (8) in here, we find that in the case of infinite resolution $[W(l \theta)=1]$, the variance of the lensed temperature map coincides with that of the unlensed map. Thus, as expected, lensing conserves the total power associated with the temperature fluctuations. This is consistent with our basic expectation that lensing only results in a transfer of power from large angular scales to small angular scales. With finite resolution at levels considered here, the variance of the lensed temperature field differs from that of the unlensed field by a few percent at most.

We will now discuss higher-order correlations of temperature due to gravitational lensing. We consider first contributions due to lensing alone, and then discuss additional contributions created by lensing-secondary correlations.

\section{A. Lensing correlations}

We will first discuss the temperature bispectrum and show that it is zero in the absence of secondary anisotropies. To understand why there is no contribution to the bispectrum, consider the moments involving three temperature terms in Fourier space:

$$
\begin{aligned}
\left\langle\widetilde{\Theta}\left(\mathbf{l}_{1}\right) \widetilde{\Theta}\left(\mathbf{l}_{2}\right) \widetilde{\Theta}\left(\mathbf{l}_{3}\right)\right\rangle_{c}= & \left\langle\left(\Theta\left(\mathbf{l}_{1}\right)-\int \frac{d^{2} \mathbf{l}_{1}^{\prime}}{(2 \pi)^{2}} \Theta\left(\mathbf{l}_{1}{ }^{\prime}\right) L\left(\mathbf{l}_{1}, \mathbf{l}_{1}{ }^{\prime}\right)\right)\left(\Theta\left(\mathbf{l}_{2}\right)-\int \frac{d^{2} \mathbf{l}_{2}^{\prime}}{(2 \pi)^{2}} \Theta\left(\mathbf{l}_{2}{ }^{\prime}\right) L\left(\mathbf{l}_{2}, \mathbf{l}_{2}{ }^{\prime}\right)\right)\right. \\
& \left.\times\left(\Theta\left(\mathbf{l}_{3}\right)-\int \frac{d^{2} \mathbf{l}_{3}^{\prime}}{(2 \pi)^{2}} \Theta\left(\mathbf{l}_{3}{ }^{\prime}\right) L\left(\mathbf{l}_{3}, \mathbf{l}_{3}{ }^{\prime}\right)\right)\right\rangle \\
= & \left\langle\Theta\left(\mathbf{l}_{1}\right) \Theta\left(\mathbf{l}_{2}\right) \Theta\left(\mathbf{l}_{3}\right)\right\rangle-\left\langle\Theta\left(\mathbf{l}_{1}\right) \Theta\left(\mathbf{l}_{2}\right)\left(\int \frac{d^{2} \mathbf{l}_{3}^{\prime}}{(2 \pi)^{2}} \Theta\left(\mathbf{l}_{3}{ }^{\prime}\right) L\left(\mathbf{l}_{3}, \mathbf{l}_{3}{ }^{\prime}\right)\right)\right\rangle \\
& +\operatorname{Perm} .+\left\langle\Theta\left(\mathbf{l}_{1}\right)\left(\int \frac{d^{2} \mathbf{l}_{2}^{\prime}}{(2 \pi)^{2}} \Theta\left(\mathbf{l}_{2}{ }^{\prime}\right) L\left(\mathbf{l}_{2}, \mathbf{l}_{2}{ }^{\prime}\right)\right)\left(\int \frac{d^{2} \mathbf{l}_{3}^{\prime}}{(2 \pi)^{2}} \Theta\left(\mathbf{l}_{3}{ }^{\prime}\right) L\left(\mathbf{l}_{3}, \mathbf{l}_{3}{ }^{\prime}\right)\right)\right\rangle+\operatorname{Perm} . \\
& -\left\langle\left(\int \frac{d^{2} \mathbf{l}_{1}^{\prime}}{(2 \pi)^{2}} \Theta\left(\mathbf{l}_{1}{ }^{\prime}\right) L\left(\mathbf{l}_{1}, \mathbf{l}_{1}{ }^{\prime}\right)\right)\left(\int \frac{d^{2} \mathbf{l}_{2}^{\prime}}{(2 \pi)^{2}} \Theta\left(\mathbf{l}_{2}{ }^{\prime}\right) L\left(\mathbf{l}_{2}, \mathbf{l}_{2}{ }^{\prime}\right)\right)\left(\int \frac{d^{2} \mathbf{l}_{3}^{\prime}}{(2 \pi)^{2}} \Theta\left(\mathbf{l}_{3}{ }^{\prime}\right) L\left(\mathbf{l}_{3}, \mathbf{l}_{3}{ }^{\prime}\right)\right)\right\rangle .
\end{aligned}
$$

All these terms, and the necessary permutations, involve an expectation value of three primary temperature anisotropies. Under our assumption of Gaussian primary temperature fluctuations, such expectation values vanish and thus there is no contribution to the bispectrum or the skewness.

The trispectrum due to lensing alone can be calculated in a similar fashion. Introducing the power spectrum of lensing potentials, following Refs. [6,9], we obtain the CMB trispectrum due to gravitational lensing as

$$
\begin{aligned}
\widetilde{T}^{\Theta}\left(\mathbf{l}_{1}, \mathbf{l}_{2}, \mathbf{l}_{3}, \mathbf{l}_{4}\right)= & -C_{l_{3}}^{\Theta} C_{l_{4}}^{\Theta}\left\{C_{\left.\mid \mathbf{l}_{1}+\mathbf{l}_{3}\right\}}^{\phi \phi}\left[\left(\mathbf{l}_{1}+\mathbf{l}_{3}\right) \cdot \mathbf{l}_{3}\right]\left[\left(\mathbf{l}_{1}+\mathbf{l}_{3}\right) \cdot \mathbf{l}_{4}\right]\right. \\
& \left.+C_{\left|\mathbf{l}_{2}+\mathbf{l}_{3}\right|}^{\phi \phi}\left[\left(\mathbf{l}_{2}+\mathbf{l}_{3}\right) \cdot \mathbf{l}_{3}\right]\left[\left(\mathbf{l}_{2}+\mathbf{l}_{3}\right) \cdot \mathbf{l}_{4}\right]\right\} \\
& + \text { Perm. }
\end{aligned}
$$

where the permutations now contain five additional terms with the replacement of $\left(l_{3}, l_{4}\right)$ by any other pair.
We can generalize our discussion of the power spectrum, bispectrum, and trispectrum to that of the $n$-point correlation function in Fourier space. In the absence of secondary anisotropies that correlate directly with the lensing potential, the $n$-point correlation function will vanish for odd $n$ for the same reason that lensing alone did not generate a bispectrum. All such terms would involve the expectation value of an odd number of temperature fluctuations, and under the assumption of Gaussian primary anisotropies, such expectation values must vanish. This statement applies in particular to the case when measurements of non-Gaussianity are made using CMB maps which have been cleaned a priori of secondary fluctuations using information such as the nonthermal frequency dependence of these fluctuations. We will discuss the case of secondary anisotropies in the next section.

The lowest even $n$th correlator after the trispectrum is the six-point correlation function in Fourier space. We can write the portion of the connected part of this correlation function containing the lowest-order contribution in $\phi$ as 


$$
\begin{aligned}
\left\langle\widetilde{\Theta}\left(\mathbf{l}_{1}\right) \ldots \widetilde{\Theta}\left(\mathbf{l}_{6}\right)\right\rangle_{c}= & \left\langle\left(\Theta\left(\mathbf{l}_{1}\right)-\int \frac{d^{2} \mathbf{l}_{1}^{\prime}}{(2 \pi)^{2}} \Theta\left(\mathbf{l}_{1}{ }^{\prime}\right) L\left(\mathbf{l}_{1}, \mathbf{l}_{1}{ }^{\prime}\right)\right) \ldots\left(\Theta\left(\mathbf{l}_{3}\right)-\int \frac{d^{2} \mathbf{l}_{3}^{\prime}}{(2 \pi)^{2}} \Theta\left(\mathbf{l}_{3}{ }^{\prime}\right) L\left(\mathbf{l}_{3}, \mathbf{l}_{3}{ }^{\prime}\right)\right) \Theta\left(\mathbf{l}_{4}\right) \Theta\left(\mathbf{l}_{5}\right) \Theta\left(\mathbf{l}_{6}\right)\right\rangle \\
& + \text { Perm. }
\end{aligned}
$$$$
=-\left\langle\int \frac{d^{2} \mathbf{l}_{1}^{\prime}}{(2 \pi)^{2}} \Theta\left(\mathbf{l}_{1}{ }^{\prime}\right) L\left(\mathbf{l}_{1}, \mathbf{l}_{1}{ }^{\prime}\right) \ldots \int \frac{d^{2} \mathbf{l}_{3}^{\prime}}{(2 \pi)^{2}} \Theta\left(\mathbf{l}_{3}{ }^{\prime}\right) L\left(\mathbf{l}_{3}, \mathbf{l}_{3}{ }^{\prime}\right) \Theta\left(\mathbf{l}_{4}\right) \Theta\left(\mathbf{l}_{5}\right) \Theta\left(\mathbf{l}_{6}\right)\right\rangle+\text { Perm. }
$$

Simplifying further, we see that the lowest order contribution in $\phi$ thus involves

$$
\begin{aligned}
\left\langle\widetilde{\Theta}\left(\mathbf{l}_{1}\right)\right. & \left.\ldots \widetilde{\Theta}\left(\mathbf{l}_{6}\right)\right\rangle_{c} \\
= & C_{l_{4}}^{\Theta} C_{l_{5}}^{\Theta} C_{l_{6}}^{\Theta}\left\langle\phi\left(\mathbf{l}_{1}+\mathbf{l}_{4}\right) \phi\left(\mathbf{l}_{2}+\mathbf{l}_{5}\right) \phi\left(\mathbf{l}_{3}+\mathbf{l}_{6}\right)\right\rangle\left[\left(\mathbf{l}_{1}+\mathbf{l}_{4}\right) \cdot \mathbf{l}_{4}\right] \\
& \times\left[\left(\mathbf{l}_{2}+\mathbf{l}_{5}\right) \cdot \mathbf{l}_{5}\right]\left[\left(\mathbf{l}_{3}+\mathbf{l}_{6}\right) \cdot \mathbf{l}_{6}\right]+\text { Perm. }
\end{aligned}
$$

The connected part of the six-point correlation function in Fourier space is thus proportional to the bispectrum of lensing potentials. We can write

$$
\begin{aligned}
& \widetilde{T}_{6}^{\Theta}\left(\mathbf{l}_{1}, \mathbf{l}_{2}, \mathbf{l}_{3}, \mathbf{l}_{4}, \mathbf{l}_{5}, \mathbf{l}_{6}\right) \\
&=C_{l_{4}}^{\Theta} C_{l_{5}}^{\Theta} C_{l_{6}}^{\Theta}\left[B^{\phi}\left(\mathbf{l}_{1}+\mathbf{l}_{4}, \mathbf{l}_{2}+\mathbf{l}_{5}, \mathbf{l}_{3}+\mathbf{l}_{6}\right)\left[\left(\mathbf{l}_{1}+\mathbf{l}_{4}\right) \cdot \mathbf{l}_{4}\right]\right. \\
&\left.\times\left[\left(\mathbf{l}_{2}+\mathbf{l}_{5}\right) \cdot \mathbf{l}_{5}\right]\left[\left(\mathbf{l}_{3}+\mathbf{l}_{6}\right) \cdot \mathbf{l}_{6}\right]\right]+ \text { Perm. }
\end{aligned}
$$

There are in total 120 such terms appearing in the six-point correlator when we include all permutations, coming from the 20 different triplets $\left(l_{i}, l_{j}, l_{k}\right)$ and the 6 permutations of each triplet.

We can generalize these derivations to the $n$-point temperature correlation in Fourier space under gravitational lensing. In the following, note that contributions to $n$-point temperature correlations in Fourier space come from $(n / 2)$-point correlations in the lensing potential. We can thus write the connected part of the $n$-point temperature correlator, when $n>2$, as

$$
\begin{aligned}
\widetilde{T}_{n}^{\Theta}\left(\mathbf{l}_{1}, \ldots, \mathbf{l}_{n}\right) & \\
= & C_{l_{n / 2+1}^{\Theta}}^{\Theta} \ldots C_{l_{n}}^{\Theta}\left[T_{n / 2}^{\phi}\left(\mathbf{l}_{1}+\mathbf{l}_{(n / 2)+1}, \ldots, \mathbf{l}_{n / 2}+\mathbf{l}_{n}\right)\right. \\
& \left.\times\left(\mathbf{l}_{1}+\mathbf{l}_{(n / 2)+1}\right) \cdot \mathbf{l}_{(n / 2)+1} \ldots\left(\mathbf{l}_{n / 2}+\mathbf{l}_{n}\right) \cdot \mathbf{l}_{n}\right]+ \text { Perm. },
\end{aligned}
$$

where $T_{n}^{\phi}\left(\mathbf{I}_{1}, \ldots, \mathbf{l}_{n}\right)$ is the $n$-point correlator of the lensing potential in Fourier space. The permutations here now involve $n ! /(n / 2)$ ! terms corresponding to the replacement of $\left(l_{(n / 2)+1}, \ldots, l_{n}\right)$ with one of the other $n ! /[(n / 2) !(n / 2) !]$ combinations and the $(n / 2)$ ! permutations of each combination. As we have discussed, note that $\widetilde{T}_{n}^{\Theta}\left(\mathbf{l}_{1}, \ldots, \mathbf{l}_{n}\right)=0$ when $n$ is odd.

In the limit that the lensing potentials are Gaussian distributed, $T_{n}^{\phi}\left(\mathbf{l}_{1}, \ldots, \mathbf{l}_{n}\right)=0$ when $n>2$. Thus, lensing of $\mathrm{CMB}$ anisotropies can only generate a trispectrum and, with smoothing, a kurtosis. The non-Gaussianity associated with the large-scale structure, however, will induce non-Gaussian contributions to the distribution of projected potentials such that $T_{n}^{\phi}\left(\mathbf{l}_{1}, \ldots, \mathbf{l}_{n}\right) \neq 0$ for some $n$. Since large-scale structure most efficiently lenses the CMB at redshifts close to 3, where the non-Gaussianity is mild, we ignore the higherorder correlations of lensing potentials and only consider the dominant power spectrum, $C_{l}^{\phi \phi}$, which contributes to the trispectrum only.

Although theoretical predictions are made in terms of ensemble-averaged correlation functions, observationally we have access to only one realization of the $\mathrm{CMB}$ and one realization of the large-scale structure. The arbitrariness of the observed realization of the large-scale structure induces additional cosmic variance beyond that normally associated with the surface of last-scatter. One consequence is that when measured on a small patch of the sky, the observed two-point correlation function of the lensed map is more anisotropic than that of the unlensed map, though isotropy holds when a sufficiently large region of the sky is considered. The excess anisotropy is induced by cosmic shear, and allows us to reconstruct the lensing deflection angle from quadratic maps involving the $\mathrm{CMB}$ temperature and polarization [9]. While we emphasize one-point statistics in this paper, a more detailed account of how higher-order statistics probe the local anisotropy induced by lensing may prove fruitful in the future.

\section{B. Lensing-secondary correlations}

The above discussion applies to the case where other secondary fluctuations do not contribute to temperature anisotropies. In practice, such a situation can be achieved when thermal CMB fluctuations are separated from dominant secondary effects like the SZ contribution. In experiments where this is not possible, say due to a lack of multifrequency data, additional non-Gaussianities will be present in the CMB map due to correlations between lensing potentials and the secondary anisotropies. The most significant of these contributions is to the three-point correlation function. We can calculate this by replacing the $\widetilde{\Theta}(\mathbf{l})$ terms in Eq. (10) with $\Theta^{t}(\mathbf{l})$. By assumption, Gaussian instrumental noise cannot generate a bispectrum, and as shown above neither does lensing alone. The total observed bispectrum is therefore that due to lensing-secondary correlations $[4,6]$

$$
B^{\Theta \mathrm{t}}\left(\mathbf{l}_{1}, \mathbf{l}_{2}, \mathbf{l}_{3}\right)=-C_{l_{1}}^{\phi s}\left[C_{l_{2}}^{\Theta}\left(\mathbf{l}_{2} \cdot \mathbf{l}_{1}\right)+C_{l_{3}}^{\Theta}\left(\mathbf{l}_{3} \cdot \mathbf{l}_{1}\right)\right]+\text { Perm. }
$$


where permutations involve two additional terms with the replacement of $l_{1}$ with $l_{2}$ and $l_{3}$. Here, $C_{l_{1}}^{\phi \mathrm{s}}$ is the power spectrum describing correlations between secondary anisotropies and the lensing potential generated by largescale structure. These correlations were discussed in detail in Ref. [4] where it was found that the most significant correlation is the one between lensing potentials and the SZ effect. We will use this correlation in illustrating our results.

The presence of secondary effects also modifies the trispectrum and generates an additional contribution beyond the one discussed in Eq. (11). Following Ref. [9], we can write this contribution as

$$
\begin{aligned}
& T^{\Theta \mathrm{s}}\left(\mathbf{l}_{1}, \mathbf{l}_{2}, \mathbf{l}_{3}, \mathbf{l}_{4}\right) \\
&=C_{l_{3}}^{\phi \mathrm{s}} C_{l_{4}}^{\phi \mathrm{s}}\left\{C_{l_{1}}^{\Theta}\left(\mathbf{l}_{3} \cdot \mathbf{l}_{1}\right)\left(\mathbf{l}_{4} \cdot \mathbf{l}_{1}\right)+C_{l_{2}}^{\Theta}\left(\mathbf{l}_{3} \cdot \mathbf{l}_{2}\right)\left(\mathbf{l}_{4} \cdot \mathbf{l}_{2}\right)\right. \\
&+\left[\mathbf{l}_{3} \cdot\left(\mathbf{l}_{1}+\mathbf{l}_{3}\right)\right]\left[\mathbf{l}_{4} \cdot\left(\mathbf{l}_{2}+\mathbf{l}_{4}\right)\right] C_{\left|\mathbf{l}_{1}+\mathbf{l}_{3}\right|}^{\Theta}+\left[\mathbf{l}_{4} \cdot\left(\mathbf{l}_{1}+\mathbf{l}_{4}\right)\right] \\
&\left.\times\left[\mathbf{l}_{3} \cdot\left(\mathbf{l}_{2}+\mathbf{l}_{3}\right)\right] C_{\left|\mathbf{l}_{1}+\mathbf{l}_{4}\right|}^{\Theta}\right\}+ \text { Perm. }
\end{aligned}
$$

where permutations involve five additional terms involving the pairings of $\left(l_{3}, l_{4}\right)$.

Due to an increase in terms as one goes to higher order, we failed to obtain a general expression for the $n$-point correlator of temperature fluctuations in Fourier space due to lensing-secondary correlations. As we will soon discuss, cumulants beyond the skewness are unlikely to be important as we find kurtosis to be undetectable even for a perfect experi- ment with no noise and all-sky observations. We expect this to hold true even when considering higher-order moments beyond the kurtosis.

\section{SKEWNESS AND KURTOSIS}

A simple way to identify the non-Gaussianity induced in the CMB by gravitational lensing is to measure the higherorder cumulants of its one-point probability distribution function $P_{\mathrm{obs}}\left(\Theta^{\mathrm{t}} ; \theta\right)$ smoothed with beam width $\theta$. This observed one-point probability distribution function (PDF) is actually a convolution of the signal $\operatorname{PDF} P_{\text {sig }}\left(\Theta^{\text {sig }} ; \theta\right)$ with the noise PDF $P_{\text {noise }}\left(\Theta^{\mathrm{n}} ; \theta\right)$ as described below, where $\Theta^{\text {sig }}$ $=\widetilde{\Theta}+\Theta^{\mathrm{s}}$ is the total of both lensed primary and secondary contributions to the signal. The signal PDF can be expressed in terms of its cumulants, which we now proceed to calculate. The third and fourth cumulants are proportional to the dimensionless quantities known as the skewness, $S$, and the kurtosis, $K$, respectively:

$$
\begin{aligned}
& S(\theta) \equiv[\sigma(\theta)]^{-3} \int\left(\Theta^{\mathrm{sig}}\right)^{3} P_{\mathrm{sig}}\left(\Theta^{\mathrm{sig}} ; \theta\right) d \Theta^{\mathrm{sig}}, \\
& K(\theta) \equiv[\sigma(\theta)]^{-4} \int\left(\Theta^{\mathrm{sig}}\right)^{4} P_{\mathrm{sig}}\left(\Theta^{\mathrm{sig}} ; \theta\right) d \Theta^{\mathrm{sig}}-3 .
\end{aligned}
$$

They can be expressed as integrals over the bispectrum and trispectrum derived in the preceding section according to Eq. (6):

$$
\begin{aligned}
& S(\theta)=[\sigma(\theta)]^{-3} \int \frac{d^{2} \mathbf{l}_{1}}{(2 \pi)^{2}} \frac{d^{2} \mathbf{l}_{2}}{(2 \pi)^{2}} \frac{d^{2} \mathbf{l}_{3}}{(2 \pi)^{2}}(2 \pi)^{2} \delta_{\mathrm{D}}\left(\mathbf{l}_{123}\right) B^{\mathrm{t}}\left(\mathbf{l}_{1}, \mathbf{l}_{2}, \mathbf{l}_{3}\right) W\left(l_{1} \theta\right) W\left(l_{2} \theta\right) W\left(l_{3} \theta\right), \\
& K(\theta)=[\sigma(\theta)]^{-4} \int \frac{d^{2} \mathbf{l}_{1}}{(2 \pi)^{2}} \frac{d^{2} \mathbf{l}_{2}}{(2 \pi)^{2}} \frac{d^{2} \mathbf{l}_{3}}{(2 \pi)^{2}} \frac{d^{2} \mathbf{l}_{4}}{(2 \pi)^{2}}(2 \pi)^{2} \delta_{\mathrm{D}}\left(\mathbf{l}_{1234}\right) T^{\mathrm{t}}\left(\mathbf{l}_{1}, \mathbf{l}_{2}, \mathbf{l}_{3}, \mathbf{l}_{4}\right) W\left(l_{1} \theta\right) W\left(l_{2} \theta\right) W\left(l_{3} \theta\right) W\left(l_{4} \theta\right)
\end{aligned}
$$

Inserting Eqs. (16), (11), and (17) into the above expressions, and adopting a Gaussian window function $W(l \theta)=e^{-\left(l \sigma_{b}\right)^{2} / 2}$ with $\sigma_{b}=\theta / \sqrt{8 \ln 2}$, we obtain

$$
\begin{aligned}
S(\theta)= & \frac{6}{(2 \pi)^{2}[\sigma(\theta)]^{3}} \int l_{1}^{2} d l_{1} l_{2}^{2} d l_{2} C_{l_{1}}^{\Theta} C_{l_{2}}^{\phi \mathrm{s}} I_{1}\left(\sigma_{b}^{2} l_{1} l_{2}\right) e^{-\sigma_{b}^{2}\left(l_{1}^{2}+l_{2}^{2}\right)}, \\
K^{\phi \phi}(\theta)= & \frac{12}{(2 \pi)^{3}[\sigma(\theta)]^{4}} \int d l_{1} l_{1}^{3} C_{l_{1}}^{\phi \phi} e^{-\sigma_{b}^{2} l_{1}^{2}}\left[\int d l_{2} l_{2}^{2} C_{l_{2}}^{\Theta} I_{1}\left(\sigma_{b}^{2} l_{1} l_{2}\right) e^{\left.-\sigma_{b}^{2} l_{2}^{2}\right]^{2},}\right. \\
K^{\phi \mathrm{s}}(\theta)= & \frac{12}{(2 \pi)^{3}[\sigma(\theta)]^{4}} \int d l_{1} l_{1}^{3} C_{l_{1}}^{\Theta} e^{-\sigma_{b}^{2} l_{1}^{2}}\left\{\left[\int d l_{2} l_{2}^{2} C_{l_{2}}^{\phi \mathrm{s}} I_{1}\left(\sigma_{b}^{2} l_{1} l_{2}\right) e^{\left.-\sigma_{b}^{2} l_{2}^{2}\right]^{2}}\right.\right. \\
& -\frac{1}{2 \pi} \int l_{2}^{2} d l_{2} l_{3}^{2} d l_{3} d \varphi C_{l_{2}}^{\phi \mathrm{s}} C_{l_{3}}^{\phi \mathrm{s}} I_{1}\left(\sigma_{b}^{2} l_{2} \sqrt{\left.l_{1}^{2}+l_{3}^{2}+2 l_{1} l_{3} \cos \varphi\right)} e^{-\sigma_{b}^{2}\left(l_{2}^{2}+l_{3}^{2}+l_{1} l_{3} \cos \varphi\right)} \frac{l_{1} \cos \varphi+l_{3} \cos { }^{2} \varphi}{\sqrt{l_{1}^{2}+l_{3}^{2}+2 l_{1} l_{3} \cos \varphi}}\right\} .
\end{aligned}
$$


Here $I_{1}(x)$ is a modified Bessel function of the first kind.

In the presence of instrumental noise, the observed onepoint probability distribution function (PDF) will be a convolution of the signal PDF characterized by the skewness and kurtosis given above, and a Gaussian noise PDF: $P_{\text {obs }}\left(\Theta^{\mathrm{t}}\right)=\int d \tau P_{\text {sig }}(\tau) P_{\text {noise }}\left(\Theta^{\mathrm{t}}-\tau\right)$. In order to perform this convolution we must first determine the explicit form of the signal PDF that will have nonzero skewness or kurtosis, but vanishing higher cumulants. To do this, we follow the formalism discussed in Ref. [13] and references therein. The PDF of a random variable $\delta$ with zero mean and variance $\sigma^{2}$ can be expressed as a Gram-Charlier series in the normalized variable $\nu \equiv \delta / \sigma$ :

$$
p(\nu)=c_{0} \phi(\nu)+\frac{c_{1}}{1 !} \phi^{(1)}(\nu)+\frac{c_{2}}{2 !} \phi^{(2)}(\nu)+\ldots,
$$

where $\phi(\nu) \equiv(2 \pi)^{-1 / 2} e^{-\nu^{2} / 2}$ is a Gaussian distribution. The $\phi^{(l)}(\nu)$ are derivatives of the Gaussian distribution with respect to $\nu$ :

$$
\phi^{(l)}(\nu) \equiv \frac{d^{l} \phi}{d \nu^{l}}=(-1)^{l} H_{l}(\nu) \phi(\nu),
$$

and the $H_{l}(\nu)$ are Hermite polynomials with the unconventional normalization,

$$
\int_{-\infty}^{\infty} H_{l}(\nu) H_{m}(\nu) \phi(\nu) d \nu=l ! \delta_{l m} .
$$

The central moments of the PDF are defined as

$$
\mu_{l} \equiv \sigma^{l} \int_{-\infty}^{\infty} p(\nu) \nu^{l} d \nu
$$

while the cumulants or "connected" portions of these moments can be derived from the relation

$$
M_{l} \equiv \frac{d^{l} \ln \left\langle e^{t \delta}\right\rangle}{d t^{l}}
$$

Using the expansion (21) and the orthogonality relation (23), the coefficients of the Gram-Charlier series can be expressed in terms of the central moments. By inverting Eq. (25), the central moments can then be reexpressed in terms of cumulants. As discussed in the previous section, the assumption that the lensing potential is Gaussian implies that all cumulants of higher order than the kurtosis must vanish. Using this result, we can rewrite the Gram-Charlier expansion as a power series in the skewness $S$ or kurtosis $K$, which in the case of lensing will be small quantities,

$$
\begin{aligned}
& p(\nu)=\left[1+\frac{1}{3 !} S H_{3}(\nu)+\frac{10}{6 !} S^{2} H_{6}(\nu)+\ldots\right] \phi(\nu), \\
& p(\nu)=\left[1+\frac{1}{4 !} K H_{4}(\nu)+\frac{35}{8 !} K^{2} H_{8}(\nu)+\ldots\right] \phi(\nu) .
\end{aligned}
$$

These power series can be convolved with a Gaussian noise PDF of variance $\sigma_{\text {noise }}^{2}(\theta)$ to obtain the observed PDF $P_{\text {obs }}\left(\Theta^{t}\right)$. To linear order in the true skewness and kurtosis, we find

$$
S_{\text {obs }}(\theta)=S(\theta)\left\{\frac{\sigma^{2}(\theta)}{\sigma^{2}(\theta)+\sigma_{\text {noise }}^{2}(\theta)}\right\}^{3 / 2},
$$

$K_{\text {obs }}(\theta)$

$$
=\frac{K(\theta)}{8}\left\{\frac{5 \sigma^{4}(\theta)-3 \sigma_{\text {noise }}^{4}(\theta)-6 \sigma^{2}(\theta) \sigma_{\text {noise }}^{2}(\theta)}{\left[\sigma^{2}(\theta)+\sigma_{\text {noise }}^{2}(\theta)\right]^{2}}+3\right\} .
$$

As expected, the observed skewness and kurtosis converge to the signal values in the absence of noise and to zero in the case when the Gaussian noise is dominant. To actually observe skewness or kurtosis in an experimental sky map, we must construct estimators for these quantities using our data points, the $N=4 \pi f_{\text {sky }} / \pi(\theta / 2)^{2}$ pixels in the map. We can write estimators for the skewness and kurtosis as

$$
\begin{aligned}
\widehat{S \sigma}^{3} & \equiv \frac{1}{N} \sum_{i=1}^{N}\left(x_{i}-\bar{x}\right)^{3}, \\
\widehat{K \sigma}^{4} & \equiv \frac{1}{N} \sum_{i=1}^{N}\left(x_{i}-\bar{x}\right)^{4}-3\left[\frac{1}{N} \sum_{i=1}^{N}\left(x_{i}-\bar{x}\right)^{2}\right]^{2},
\end{aligned}
$$

where $\bar{x}=(1 / N) \sum_{i=1}^{N} x_{i}$ is the traditional estimator for the mean of a distribution. For a distribution like that of the CMB anisotropies which is a priori defined to have a zero mean, we find

$$
\begin{aligned}
& \left\langle{\widehat{S \sigma^{3}}}^{3}\right\rangle=\left(1-\frac{3}{N}+\frac{2}{N^{2}}\right) S \sigma^{3}, \\
& \left\langle\widehat{K \sigma}^{4}\right\rangle=\left(1-\frac{7}{N}+\frac{12}{N^{2}}-\frac{6}{N^{3}}\right) K \sigma^{4}-\frac{6}{N}\left(1-\frac{1}{N}\right) \sigma^{4} .
\end{aligned}
$$

These are biased estimators, as has been noted elsewhere under a different context [14], but in the large- $N$ limit they converge to the desired quantities. Assuming that the underlying PDF is Gaussian, the variance of these estimators to lowest order in $1 / N$ is given by

$$
\begin{gathered}
\sigma_{\widehat{S \sigma}^{3}}^{2} \equiv\left\langle\left(\widehat{S \sigma}^{3}\right)^{2}\right\rangle-\left\langle\widehat{S \sigma}^{3}\right\rangle^{2}=\frac{3 !}{N} \sigma^{3}, \\
\sigma_{\widehat{K \sigma}}^{2} \equiv\left\langle\left(\widehat{K \sigma}^{4}\right)^{2}\right\rangle-\left\langle\widehat{K \sigma}^{4}\right\rangle^{2}=\frac{4 !}{N} \sigma^{4} .
\end{gathered}
$$

An alternate derivation of these variances can be obtained from the explicit form of the PDFs following Eq. (26). If $N$ pixels or data points are collected and binned such that $p_{i}$ is the probability that a data point will fall within bin $i$ and $\sigma_{i}$ 

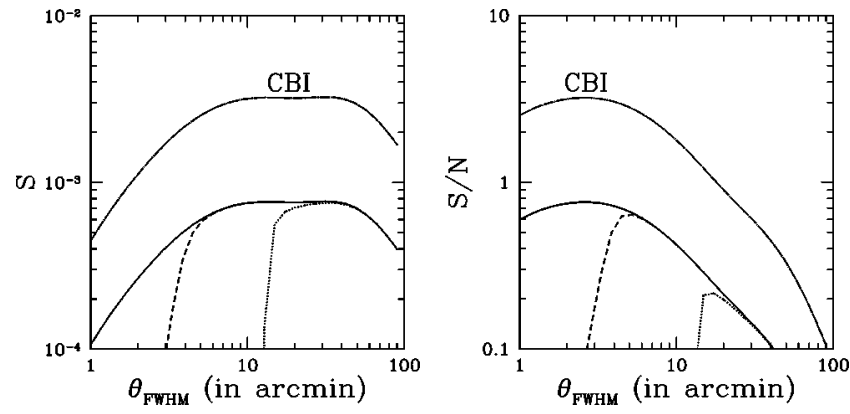

FIG. 1. Left: The skewness due to lensing-SZ correlations for a perfect (no-noise) experiment (solid line), Planck (dashed line), and MAP (dotted line) for $\sigma_{8}=0.9$. The CBI $1 \sigma$ upper bound of $\sigma_{8}$ $\leqslant 1.2$ leads to a higher value for the skewness as indicated by the dot-dashed line. Right: The signal-to-noise ratio for the detection of skewness in CMB data with curves labeled as in the left figure. We assume full sky coverage; for partial sky coverage the signal-tonoise ratio scales as $\sqrt{f_{\text {sky }}}$, where $f_{\text {sky }}$ is the fraction of sky covered.

is the standard deviation of that probability, then the best variance of a parameter $\epsilon$ characterizing the PDF is given by the Cramér-Rao bound [15],

$$
\frac{1}{\sigma_{\epsilon}^{2}}=\sum_{i}\left(\frac{\partial p_{i}}{\partial \epsilon}\right)^{2} \frac{1}{\sigma_{i}^{2}}
$$

If the error on each bin is assumed to be Poisson, then $\sigma_{i}^{2}$ $=p_{i} / N$. In the limit of a continuous PDF, $p_{i} \rightarrow p(\nu) d \nu$ and the discrete sum (31) becomes an integral

$$
\frac{1}{\sigma_{\epsilon}^{2}}=N \int\left(\frac{\partial p}{\partial \epsilon}\right)^{2} p^{-1} d \nu
$$

Inserting Eq. (26) into Eq. (32) under the Gaussian null hypothesis $S=K=0$, we find lowest attainable errors as $\sigma_{\epsilon}^{2}$ $=3 ! / N, 4 ! / N$ for $\epsilon=S, K$ in agreement with the explicit calculation of the variance of our estimators noted in Eq. (30). Further discussion of the variance associated with different estimators for the skewness and kurtosis is included in the Appendix.

\section{RESULTS AND DISCUSSION}

\section{A. Skewness}

We illustrate in Fig. 1 our results for skewness due to the correlation between lensing and the SZ effect. We calculate this correlation following Ref. [17] using the halo approach to large-scale structure [18]. The skewness approaches zero at small values of the smoothing scale, consistent with our conclusion that no non-Gaussian signatures exist in the PDF in the limit of infinite resolution. As shown, skewness due to the lensing-SZ correlation peaks at an angular scale of tens of arcminutes, which is in the range of interest to upcoming experiments such as MAP and Planck. When calculating expected signal-to-noise ratios for these experiments, we use detector sensitivities and resolutions tabulated in Ref. [16]. For simplicity, we combine information from individual fre- quency channels to form one estimate of temperature with an overall noise given by inversely weighting individual noise contributions.

The skewness as shown has signal-to-noise ratios slightly less than unity suggesting that its detection may be hard and potentially affected by noise. However, recent small-scale excess-power detections by experiments such as CBI [19] raise the possibility that we may have underestimated the lensing-SZ correlation and thus the skewness. The lensing-SZ power spectrum $C_{l}^{\phi s}$ is roughly proportional to the fifth power of $\sigma_{8}$, the standard deviation of linear mass fluctuations within an $8 h^{-1} \mathrm{Mpc}$ sphere. If we adopt the CBI $1 \sigma$ upper bound of $\sigma_{8} \leqslant 1.2$ [19] as opposed to the value $\sigma_{8}=0.9$ suggested by previous studies, our signal increases by a factor of 4.21. In this case, Planck could conceivably detect skewness with a signal-to-noise ratio of 2.5. The potential for detection of the temperature skewness is consistent with previous expectations that the temperature anisotropy bispectrum due to lensing-SZ correlation can be detected in future data [4]. The cumulative signal-to-noise ratio for skewness, however, is significantly smaller than that for the full bispectrum because the skewness is a single number while the bispectrum contains all information related to non-Gaussianities at the three-point level. As described below, we find a similar reduction in the signal-to-noise ratio for kurtosis when compared to the full trispectrum.

The frequency dependence of the SZ effect allows us to construct an SZ map of the sky as well as a temperature map with the SZ effect removed. This provides us a unique opportunity to test our understanding of non-Gaussianity at the three-point level. If skewness is purely a consequence of lensing-SZ correlations as posited in this paper, then the skewness obtained by combining one measurement of the SZ map with two measurements of the SZ-cleaned temperature map at the same location using the estimator in Eq. (28) should be precisely one-third that produced by three measurements of the total anisotropy map. This corresponds to the fact that the composite map will sample only one of the three permutations appearing in Eq. (16).

\section{B. Kurtosis}

Both lensing kurtosis $K^{\phi \phi}$ and the kurtosis $K^{\phi s}$ due to lensing-SZ correlations are undetectable even for a perfect no-noise experiment as illustrated in Fig. 2. Since the cumulative signal-to-noise ratio for $K^{\phi s}$ is well below one, we expect it to remain undetectable despite any uncertainty in our calculation of the SZ effect. Note our prediction of the lensing kurtosis $K^{\phi \phi}$ is likely to be more certain since it only depends on the matter power spectrum, with contributions coming mainly from the linear regime. Thus, uncertainties in non-linear aspects of clustering are unlikely to affect our conclusion.

The signal-to-noise value for $K^{\phi \phi}$ can be compared to the cumulative signal-to-noise ratio for the direct detection of the full trispectrum due to lensing, which in the case of Planck can be as high as $\sim 55$ [6]. Consequently, although the lensing kurtosis cannot be detected directly from the data, lensing effects associated with this kurtosis can be used 

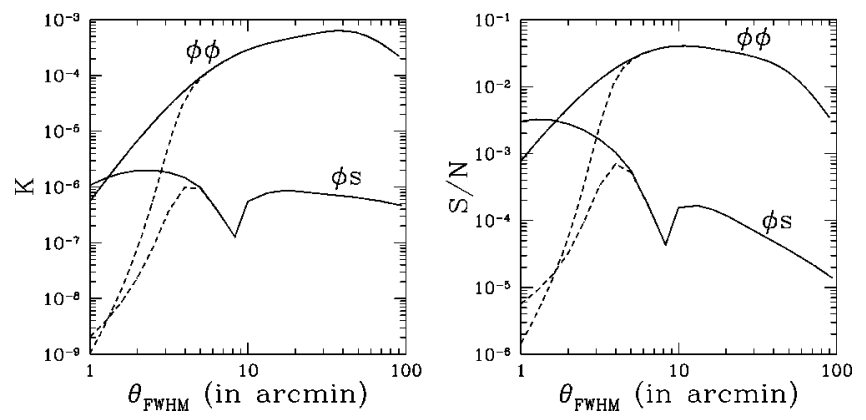

FIG. 2. Left: The kurtosis $K^{\phi \phi}$ due to lensing autocorrelations and $K^{\phi s}$ due to lensing-SZ cross-correlations for a perfect (nonoise) experiment (solid line) and Planck (dashed line). The kurtosis due to lensing-SZ correlations is negative at smoothing scales below the kink at $\sim 8$ arcmin and positive thereafter; its absolute value is shown here. Right: The signal-to-noise ratio for the detection of kurtosis in CMB data with curves labeled as in the left figure. We assume full sky coverage; for partial sky coverage the signal-to-noise ratio scales as $\sqrt{f_{\text {sky }}}$, where $f_{\text {sky }}$ is the fraction of sky covered.

to reconstruct the lensing deflection angle as described in Refs. [8,9], again with cumulative signal-to-noise ratios significantly greater than that for the kurtosis itself. The higher signal-to-noise ratio in lensing reconstruction is possible for two reasons. Unlike the kurtosis, which averages indiscriminately over all configurations of the trispectrum as shown in Eq. (19), lensing reconstruction is sensitive to certain configurations of the trispectrum, mainly those that contribute to the power spectrum of squared temperature. This avoids severe positive-negative cancellations that significantly reduce the signature of non-Gaussianity. Secondly, the noise contribution associated with lensing reconstruction is also a priori reduced through a filter which is designed to extract information on the lensing potentials optimally.

The low signal-to-noise ratio associated with the kurtosis is also consistent with the fact that real-space moments, in general, suffer from excess noise. Though such statistics are easily measurable in data, they do not provide the most optimal methods to search for the existence of non-Gaussian signatures. While we recommend construction of cumulants such as skewness and kurtosis as a first step in understanding non-Gaussianity from effects such as lensing, we suggest that full measures of quantities such as bispectrum and trispectrum will be necessary to fully understand the nonGaussian behavior of lensing. If measurement of such statistics are still cumbersome, we suggest the use of quadratic statistics in real space, such as the squared-temperaturetemperature [7] and the squared-temperature-squaredtemperature [9] power spectra which probe certain configurations of the bispectrum and trispectrum.

\section{ACKNOWLEDGMENTS}

This work was supported in part by NSF AST-0096023, NASA NAG5-8506, and DoE DE-FG03-92-ER40701. Kesden acknowledges the support of the NSF Graduate program, and A.C. acknowledges support from the Sherman Fairchild Foundation.

\section{APPENDIX: VARIANCE OF SKEWNESS AND KURTOSIS ESTIMATORS}

A question arose during the composition of this paper as to the appropriate variance for estimates of the skewness and kurtosis of a Gaussian distribution. The true skewness and kurtosis of a Gaussian distribution are necessarily zero, but given $N$ data points $x_{i}$ drawn from this distribution even unbiased estimators will yield results distributed about zero with some variance. Some sources (e.g., [20]) indicate variances of $15 / N$ and $96 / N$, respectively, for the skewness and kurtosis estimators defined in Eq. (28) as opposed to our values of $6 / N$ and $24 / N$. This discrepancy prompted us to investigate further. The estimators of Eq. (28) differ from those given in Ref. [20] in that they are estimators for the third and fourth cumulants rather than the dimensionless skewness and kurtosis to which they are proportional. Assuming an underlying Gaussian distribution with a variance of unity, standard propagation of errors reveals that the two pairs of estimators have the same variances to lowest order in $1 / N$. However, the naive estimators

$$
\begin{aligned}
& \widehat{S \sigma}^{3 \prime} \equiv \frac{1}{N} \sum_{i=1}^{N} x_{i}^{3}, \\
& \widehat{K \sigma}^{4 \prime} \equiv \frac{1}{N} \sum_{i=1}^{N} x_{i}^{4}-3\left[\frac{1}{N} \sum_{i=1}^{N} x_{i}^{2}\right]^{2}
\end{aligned}
$$

do indeed have variances of $15 / N$ and $96 / N$ for skewness and kurtosis, respectively. We show this explicitly for the naive skewness estimator $\widehat{S \sigma^{3}}$. The ensemble average of this estimator is simply $S \sigma^{3}$ so it is truly an unbiased estimator for the skewness. However, taking the ensemble average of $\left(\widehat{S \sigma^{3 \prime}}\right)^{2}$ we find

$$
\left\langle\left(\widehat{S \sigma^{3}}\right)^{2}\right\rangle=\frac{1}{N}\left[\mu_{6}+(N-1) S^{2} \sigma^{6}\right]
$$

leading to a variance

$$
\sigma_{S \sigma^{3}}^{2} \equiv\left\langle\left(\widehat{S \sigma^{3}}\right)^{2}\right\rangle-\left\langle\widehat{S \sigma^{3} \prime}\right\rangle^{2}=\frac{1}{N}\left(\mu_{6}-S^{2} \sigma^{6}\right) .
$$

For a Gaussian distribution, $\mu_{6}=15 \sigma^{6}$ and $S=0$, implying that this estimator measures skewness with a variance of $15 / N$ and is therefore less sensitive than $\widehat{S \sigma^{3}}$ defined in Eq. (28) which was shown to have a variance of $6 / N$. An entirely analogous calculation shows that the naive kurtosis estimator in Eq. (A1) has a variance of $96 / N$, not $24 / N$. 
Why do the estimators of Eq. (28) outperform those of Eq. (A1)? Although the true mean of the underlying Gaussian distribution has been chosen to be zero, the estimated mean $\bar{x}=(1 / N) \sum_{i=1}^{N} x_{i}$ of $N$ data points will not necessarily vanish. The more sophisticated estimators of Eq. (28) take this into account by subtracting the estimated mean from each data point, and are therefore able to provide lowervariance estimates of the skewness and kurtosis. These lower values for the variances are adopted for all results concerning signal-to-noise ratios mentioned in this paper.
[1] A. Blanchard and J. Schneider, Astron. Astrophys. 184, 1 (1987); A. Kashlinsky, Astrophys. J. Lett. 331, L1 (1988); E.V. Linder, Astron. Astrophys. 206, 1999 (1988); L. Cayon, E. Martinez-Gonzalez, and J. Sanz, Astrophys. J. 413, 10 (1993); U. Seljak, ibid. 463, 1 (1996).

[2] W. Hu, Phys. Rev. D 62, 043007 (2000).

[3] See, for example, A. Cooray, "2001 Coral Gables Conference: Cosmology and Elementary Particle Physics," edited by B. Kursunoglu, Stephan L. Mintz, and A. Perlmutter, AIP Conf. Proc. No. 624, (AIP, Melville, NY, 2002), astro-ph/0203048.

[4] D.M. Goldberg and D.N. Spergel, Phys. Rev. D 59, 103002 (1999); M. Zaldarriaga and U. Seljak, ibid. 59, 123507 (1999); H.V. Peiris and D.N. Spergel, Astrophys. J. 540, 605 (2000); A. Cooray and W. Hu, ibid. 534, 533 (2000).

[5] F. Bernardeau, Astron. Astrophys. 324, 15 (1997).

[6] M. Zaldarriaga, Phys. Rev. D 62, 063510 (2000); W. Hu, ibid. 64, 083005 (2001).

[7] A. Cooray, Phys. Rev. D 65, 063512 (2002).

[8] W. Hu, Astrophys. J. 557, 79 (2001); K. Benabed, F. Bernardeau, and L. van Waerbeke, Phys. Rev. D 63, 043501 (2001); W. Hu and T. Okamoto, Astrophys. J. 574, 566 (2002).

[9] A. Cooray and M. Kesden, astro-ph/0204068.

[10] G. Hinshaw, A.J. Banday, C.L. Bennett, K.M. Górski, and A.
Kogut, Astrophys. J. 446, 67 (1995); U. Seljak and M. Zaldarriaga, Phys. Rev. Lett. 82, 2636 (1999).

[11] R.A. Sunyaev and Ya.B. Zel'dovich, Mon. Not. R. Astron. Soc. 190, 413 (1980).

[12] R.K. Sachs and A.M. Wolfe, Astrophys. J. 147, 73 (1967).

[13] R. Juszkiewicz et al., Astrophys. J. 442, 39 (1995).

[14] L. Hui and E. Gaztanaga, Astrophys. J. 519, 622 (1999).

[15] M. G. Kendall and A. Stuart, The Advanced Theory of Statistics (Griffin, London, 1969), Vol. II.

[16] A. Cooray, W. Hu, and M. Tegmark, Astrophys. J. 540, 1 (2000).

[17] A. Cooray, Phys. Rev. D 64, 043516 (2001).

[18] For a recent review, see A. Cooray and R.K. Sheth, astro-ph/0206508.

[19] B.S. Mason et al., astro-ph/0205384; A. Cooray and A. Melchiorri, Phys. Rev. D 66, 083001 (2002); E. Komatsu and U. Seljak, astro-ph/0205468.

[20] W. H. Press et al., Numerical Recipes in C: The Art of Scientific Computing (Cambridge University Press, New York, 1992). The variances of skewness and kurtosis of $15 / N$ and $96 / N$, respectively, only appear in the second edition, pp. 612 and 613. 